\title{
Should we continue breastfeeding after SARS-CoV-2 infection or mRNA vaccination?
}

\author{
Fei CHEN ${ }^{1, *}$; Chun LUAN ${ }^{1}$; Zichun WEI ${ }^{1}$; Dechen CAI ${ }^{1}$; Zhiwen CUI ${ }^{1}$; Yuyang LI $^{1}$; Hao WU ${ }^{2}$; Xiaoxia ZHANG ${ }^{1}$; XiaOli WU ${ }^{2}$ \\ ${ }^{1}$ Jining Medical University, Jining, 272000, China \\ 2 Dongping County People's Hospital, Tai-an, 271500, China
}

Key words: COVID-19, Abs, Breast milk, mRNA vaccine, Symptoms

\begin{abstract}
The coronavirus disease 2019 (COVID-19), which is caused by severe acute respiratory syndrome coronavirus 2 (SARS-CoV-2), has posed a potential threat to infant health. The World Health Organization recommended that the benefits of breastfeeding far outweigh the potential risk of transmission, but there is no denying that the current evidence is insufficient. Moreover, although the COVID-19 mRNA vaccine has played an effective role in protection against infection, individuals have increasing concerns about the safety of breastfeeding after vaccination, and which have caused some breastfeeding women to postpone vaccination or stop breastfeeding early. Thus, in this review, we provide an in-depth discussion of whether SARS-CoV-2 and the vaccine will affect babies through breast milk. On one hand, only a very small number of milk samples were identified positive for viral RNA and almost impossible to be live virus particles. The milk of most lactating women after vaccination did not contain vaccine-related mRNA and polyethylene glycol. On the other hand, the antibodies and biologically active molecules like lactoferrin are abundant in the milk of lactating women who have been infected or vaccinated, which can provide potential protection against infants' respiratory and gastrointestinal infections. Therefore, in terms of implications for clinical practice, the results of our study support that lactating women who have been infected or vaccinated should be encouraged to breastfeed their infants under the premise of taking appropriate sanitary measures.
\end{abstract}

\section{Introduction}

At present, the ongoing coronavirus disease 2019 (COVID-19) outbreak, caused by the severe acute respiratory syndrome coronavirus 2 (SARS-CoV-2), has brought severe adverse effects on global public health, society and economy. As of 18 October 2021, there are more than 230 million confirmed cases worldwide, including some 4.8 million deaths, and the number is rising sharply every day (World Health Organization, 2021). Although pediatric COVID-19 was typically mild in most cases, there were still about $10 \%$ of infants under one year of age suffering from severe COVID-19 that requires advanced care (Dong et al., 2020). More seriously, a growing number of infants seemed to exhibit signs of "multisystem inflammatory syndrome in children", which is a rare but potentially deadly inflammatory condition (Riphagen et al., 2020; Verdoni et al., 2020). Besides, infected infants can transmit SARS-CoV-2 to others (Tang et al., 2020). For these reasons, protecting these populations from infection is essential.

*Address correspondence to: Fei Chen, chenfei0336@163.com Received: 21 October 2021; Accepted: 03 December 2021
Unfortunately, none of the COVID-19 vaccines that have been vaccinated on a large scale are currently authorized or under investigation for use in infants (Pieri et al., 2021), so the protection provided by breastfeeding is particularly important for infants. However, whether the SARS-CoV-2 is present in breast milk after infection has caused widespread controversy, and people are worried that the virus may infect infants through breastfeeding. In addition, the potential transfer of the mRNA from the COVID-19 mRNA vaccine to breast milk may result in the initiation of infant immune responses and thereby alter immunocompetence. Similarly, the polyethylene glycol (PEG), which presents in the lipid film of mRNA-based vaccines, has been reported to cause an allergic reaction in rare cases (Garvey and Nasser, 2021; Sellaturay et al., 2021). These results lead to fear among lactating women, and some of them chose to stop breastfeeding. Thus, there is an urgent need to review the presence of SARS-COV-2 virus and potential allergenic components of the COVID-19 mRNA vaccine in breast milk.

Breast milk is a source of various nutrients (such as proteins and peptides) and bioactive components and which can promote neonatal growth and prevent viral and bacterial 
infections. In terms of antibodies (Abs), human milk contains a series of immunoglobulins (Igs), including IgM, secreted IgM ( $\operatorname{IgM}), \operatorname{IgA}$, secreted $\operatorname{IgA}(\operatorname{sg} \mathrm{A})$ and $\operatorname{IgG}$. Among them, $\operatorname{IgA}$ is the most abundant $\mathrm{Ab}$ isotype (about $90 \%$ of the total Igs) in human milk and plays an important protective role (Hurley and Theil, 2011). Moreover, specific IgA Abs have been detected in breast milk of mothers infected with SARS-CoV, respiratory syncytial virus and human immunodeficiency virus (Palmeira and Carneiro-Sampaio, 2016; Robertson et al., 2004). In addition, lactoferrin (LF), one of the biologically active molecules, has an antiviral effect on a variety of human viruses including rotavirus, hepatitis $\mathrm{C}$, herpes virus, etc. (van der Strate et al., 2001). Therefore, the existences and functions of these important biologically active molecules that may protect babies from COVID-19 infection in the milk from the mother infected or vaccinated are also worthy of attention.

The possible presence of SARS-CoV-2 RNA and vaccine-related $m R N A$ in breast milk

To obtain definitive information regarding the transmission risks of SARS-CoV-2 through breast milk, we summarized the detection results of human breast milk and infants in Table 1 (Chambers et al., 2020; Fenizia et al., 2020; Gao et al., 2020; Sahin et al., 2020; Bertino et al., 2020; Liu et al., 2020; Sahin et al., 2021; Chen et al., 2020; Kunjumon et al., 2021; Kilic et al., 2021). Among the 285 breast milk samples from SARS-CoV-2 positive mothers, only 9 had SARS-CoV-2 RNA detected. Importantly, the virus culture in one of the RT-PCR positive samples was negative, indicating that it might not be replicationcompetent live virus that being discovered. Moreover, 279 nasopharyngeal swabs were performed in infants, with only 15 positive results for SARS-CoV-2 (Table 1). Therefore, we speculate that the virus was primarily transmitted through respiratory droplets and contact routes. Therefore, breastfeeding has a low risk of transmitting SARS-CoV-2 and it can be continued under prudent precautions.

Low et al. (2021a) detected a very low level of vaccine mRNA using phenol-chloroform extraction, the gold standard for RNA extraction and double quencher $\mathrm{qPCR}$ probes (Low et al., 2021a). However, the discrepancies in the sensitivity of the detection method would lead to different test results. In most cases, vaccine mRNA was not detected in milk samples after vaccination (Golan et al., 2021a; Golan et al., 2021b). Notably, even if a low level of vaccine mRNA was detected, it was expected to be easily destroyed by enzymes in the infant's gut. At the same time, although allergic reactions have been reported in a few cases, no significant levels of PEG were found in breast milk after vaccination (Golan et al., 2021c). Nevertheless, lots of people are panicking about the lack of data on adverse effects of breastfeeding mothers and infants.

To explore the safety of vaccination during breastfeeding, we conducted literature review using keywords "SARS-CoV-2, lactation, breast milk, breastfed, breastfeeding, mRNA vaccine" in Pubmed, Science Direct and Google Scholar database, and made Table 2 to summarize the symptoms of breastfeeding women who received the COVID-19 mRNA vaccine (Pfizer or Moderna) and adverse events in their children (Perl et al., 2021; McLaurin-Jiang et al., 2021; Low et al., 2021a; Golan et al., 2021c; Gray et al., 2021; Jakuszko et al., 2021; Low et al., 2021b; Selma-Royo et al., 2021; Lechosa-Muñiz et al., 2021; Low et al., 2021c). In terms of the symptoms in mothers after vaccination, following Dose 1, the frequency of specific symptoms such as pain, redness, joint or muscle pain at the injection site was not differed by vaccine brands. Following Dose 2, women who received the Moderna brand vaccines were significantly more prone to experience chills, muscle/body pain, fever, and vomiting than received the Pfizer vaccine brand. Meanwhile, allergic reactions were less common in patients treated with the second dose of any COVID-19 vaccine, which is likely because an allergic reaction to the first dose led to a contraindication to receiving a subsequent dose. Furthermore, the incidences of mastitis after the first and second doses of vaccination were lower than or close to the $2.5 \%$ to $20 \%$ estimated by global mastitis epidemiology (Table 2). It was

TABLE 1

Characteristics of breast milk and infants of women infected with SARS-CoV-2

\begin{tabular}{|c|c|c|c|c|}
\hline \multicolumn{3}{|c|}{ Human milk } & \multirow{2}{*}{$\begin{array}{l}\text { Infants } \\
\text { RT-PCR positive rate of } \\
\text { nasopharyngeal swab }\end{array}$} & \multirow[t]{2}{*}{ Reference } \\
\hline RT-PCR positive rate & Viral culture & $\begin{array}{l}\text { Anti-SARS-CoV-2 IgM } \\
\text { and IgG antibodies }\end{array}$ & & \\
\hline $5.6 \%(1 / 18)$ & Negative & NP & NP & Chambers et al. (2020) \\
\hline $3.2 \%(1 / 31)$ & NP & $3.2 \%(1 / 31)$ & $6.5 \%(2 / 31)$ & Fenizia et al. (2020) \\
\hline $0 \%(0 / 12)$ & NP & $75.0 \%(3 / 4)$ & $0 \%(0 / 12)$ & Gao et al. (2020) \\
\hline $0 \%(0 / 29)$ & NP & NP & $0 \%(0 / 29)$ & Sahin et al. (2020) \\
\hline $7.1 \%(1 / 14)$ & NP & NP & $28.6 \%(4 / 14)$ & Bertino et al. (2020) \\
\hline $0 \%(0 / 10)$ & NP & NP & $0 \%(0 / 19)$ & Liu et al. (2020) \\
\hline $0.76 \%(1 / 131)$ & NP & $\mathrm{NP}$ & $0 \%(0 / 133)$ & Sahin et al. (2021) \\
\hline $0 \%(0 / 6)$ & NP & NP & $0 \%(0 / 6)$ & Chen et al. (2020) \\
\hline $5.3 \%(1 / 19)$ & NP & NP & $0 \%(0 / 19)$ & Kunjumon et al. (2021) \\
\hline $26.7 \%(4 / 15)$ & NP & NP & $56.3 \%(9 / 16)$ & Kilic et al. (2021) \\
\hline
\end{tabular}

Note: RT-PCR, Real-Time reverse-transcriptase-Polymerase-Chain-Reaction; NP, not provided. 


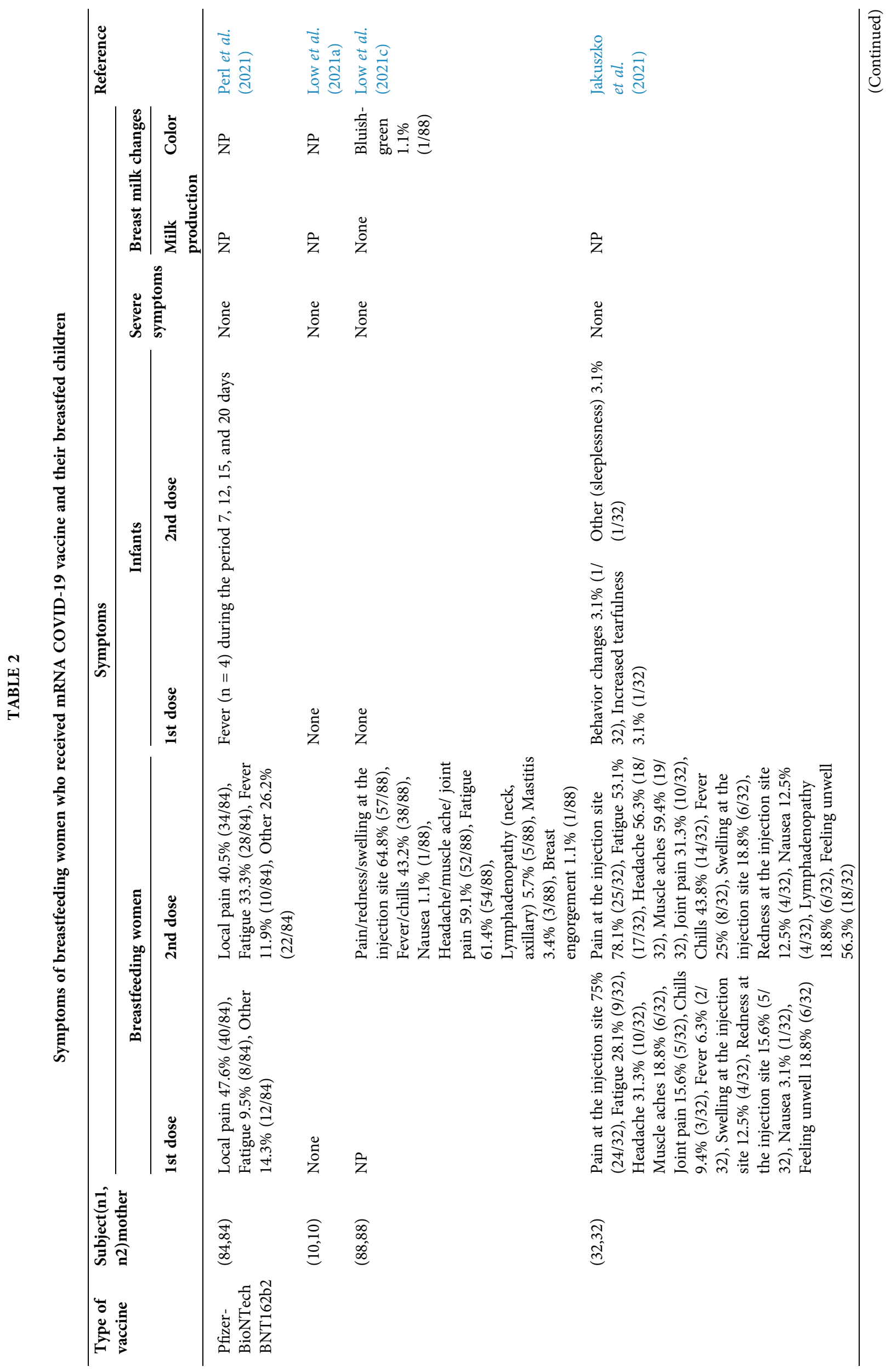




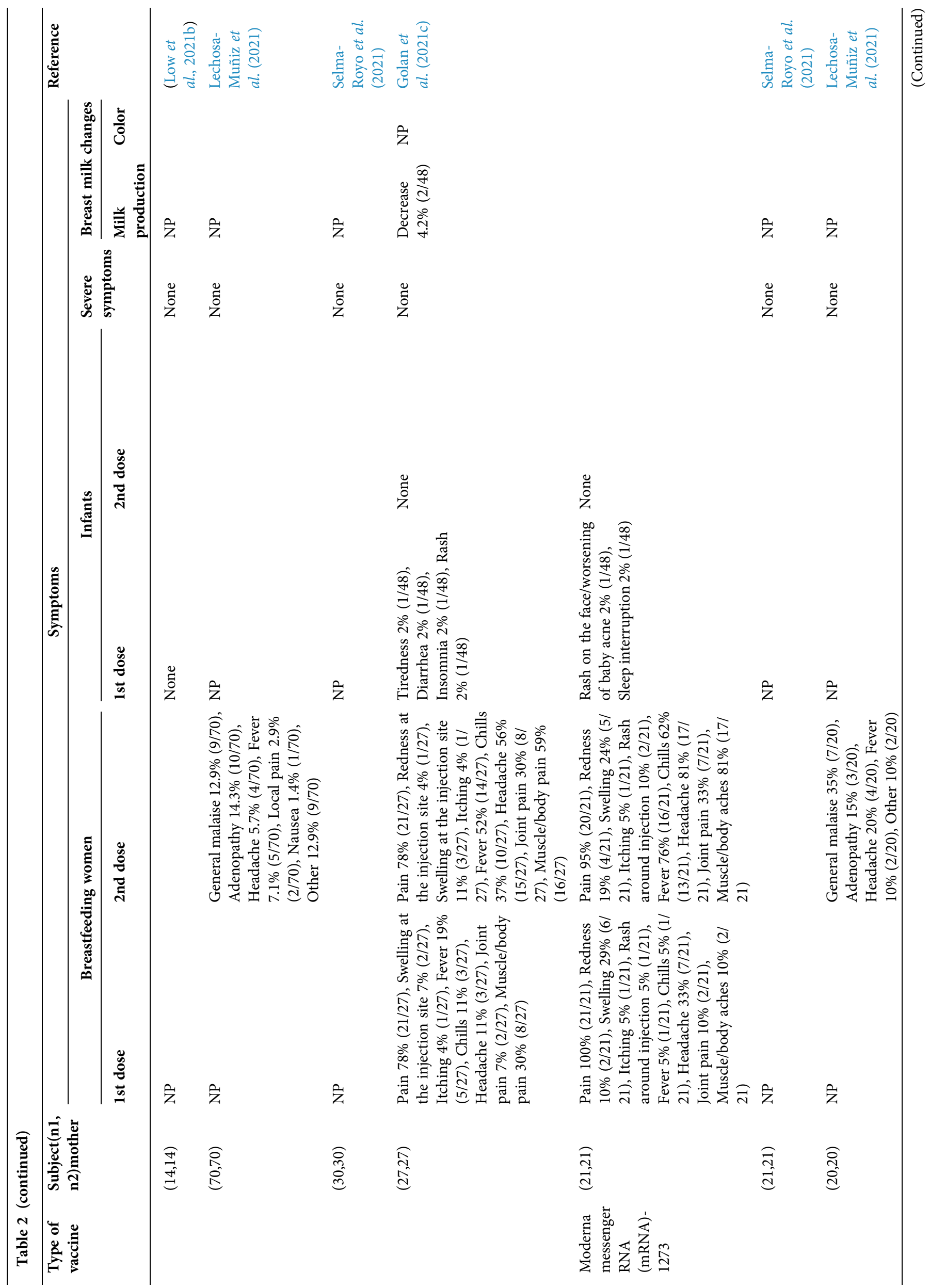




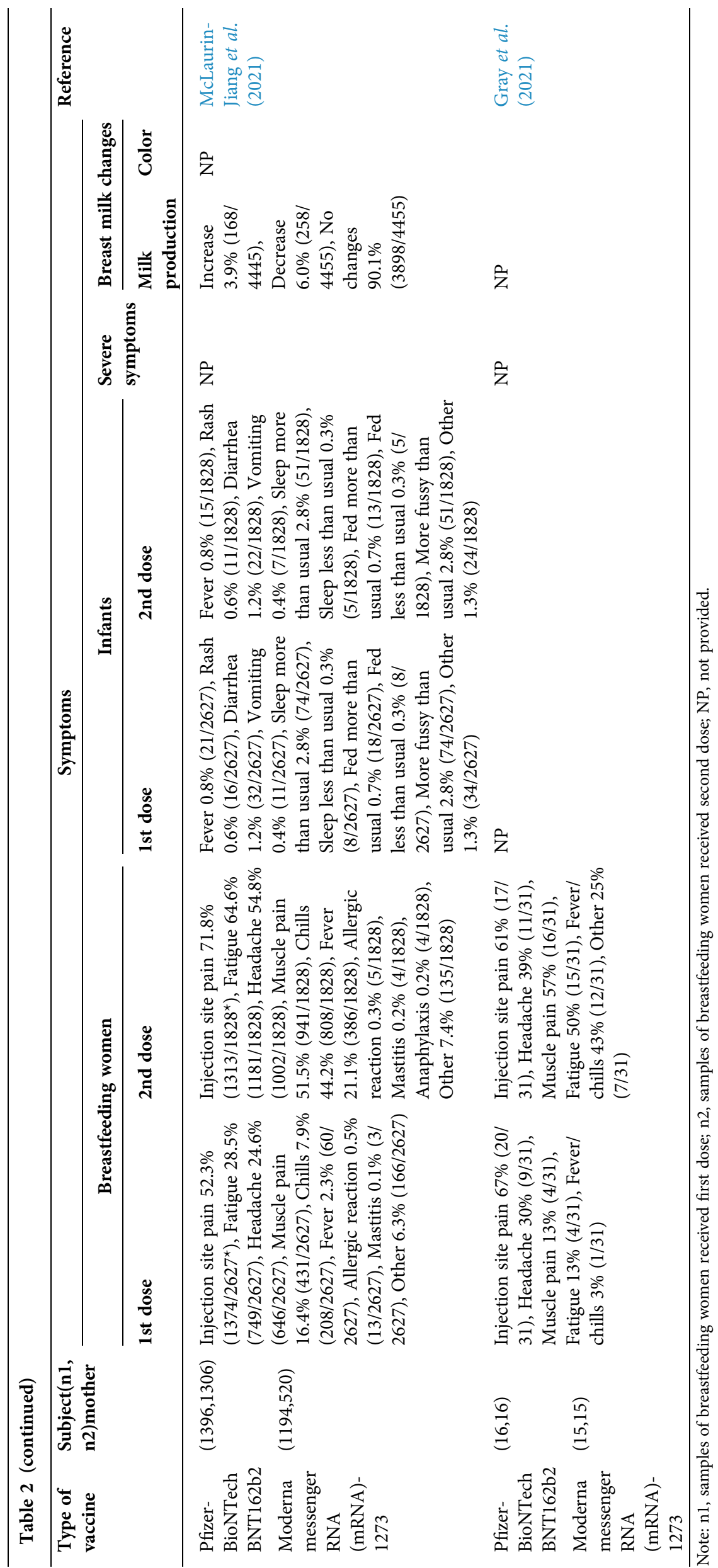


worth mentioning that the milk production of most mothers did not change after vaccination, with only a small number of mothers experiencing a mild increase or decrease in milk supply (Table 2), and all cases returned to normal within $72 \mathrm{~h}$ after receiving the vaccine without any intervention. Interestingly, one mother reported that her color of breast milk turned bluish-green within $24 \mathrm{~h}$ after receiving the first dose of the Pfizer vaccine, but the alteration might be attributed to maternal dietary changes rather than vaccination as it did not recur after the second dose vaccination (Low et al., 2021c). Additionally, the children whose mothers were vaccinated with either brand or either dose had almost no serious adverse events, and only a few cases had one or more symptoms (Table 2). Mothers who received a second vaccine dose were more likely to perceive some symptoms in their breastfed children, including irritability, lack of sleep, and restlessness. However, most of these symptoms disappeared up to $72 \mathrm{~h}$ after vaccination (Golan et al., 2021c). Four infants had fever at 7, 12, 15, and 20 days after maternal vaccination. Except for one infant who was admitted to the hospital for neonatal fever evaluation and received antibiotic treatment, all infants recovered on their own (Perl et al., 2021). Overall, no mothers or infants showed any serious adverse symptoms after vaccination. These results support the safety of vaccination during breastfeeding.

\section{Antibodies in breast milk after COVID-19 infection or mRNA vaccination}

Infection or exposure to SARS-CoV-2 can cause the production of anti-SARS-CoV-2 Abs in human milk. On one hand, sIgA has been shown to be the main form of receptor-binding domain-specific IgA in milk samples obtained from COVID-19 patients and recovered donors (Fox et al., 2020). It can prevent adhesion to target epithelial cells through binding to the SARS-CoV-2 nucleocapsid protein or neutralization of the spike protein, and thereby provide a potential protective effect against gastrointestinal infection in the infant. Of note is that the specificity of $\operatorname{sIgA}$ is determined by the maternal immune response to prior infection and this may explain the low infection rate or milder symptoms of the infants who were breastfed by SARS-CoV-2-infected mothers. On another hand, IgM and IgG are less in human milk samples collected (Table 1), but these two Abs have known immune-surveillance properties. IgG not only plays an anti-inflammatory role, but also prevents infection at the intestinal level. High-avidity IgM Abs can act as an essential part of protecting the infant's mucosal surfaces from bacteria and viruses. In addition, because the capillary bed connects gastrointestinal system with the circulatory and lymphatic systems, the lacteal lymphatic capillary located in intestinal villi of the small intestine may absorb and transport breast milk Abs to the respiratory system, thereby providing respiratory protection for newborns (Demers-Mathieu et al., 2021a). By the way, it has been reported that SARS-CoV-2-spiked donor milk samples have no cytopathic activity after pasteurization (Unger et al., 2020). Further, some have suggested that breast milk should be pasteurized. However, due to the heat load of milk exposure, Holder pasteurization can affect the immune protection provided by breast milk and reduce the immunological and biological value. Mothers should be supported and encouraged to offer fresh breast milk to their infants under the premise of applying appropriate hygiene measures.

After the 1st dose of COVID 19 mRNA vaccine, maternal plasma IgG and IgM Abs increased significantly, and the IgG level after the 2 nd dose has been proven to increase by 6 -fold (Golan et al., 2021c), which indicates the importance of the 2nd dose enhancing the antibody response. Besides, the antibody response was highly synchronized between serum and breast milk (Friedman et al., 2021). A large number of anti-SARS-CoV-2 IgA and IgG Abs were detected in breast milk beginning at Day 7 after the initial dose, and antiSARS-CoV-2 IgG dominated (Baird et al., 2021). As we all know, IgG in breast milk has been shown to play a critical role in neonatal immunity against several other viral pathogens, including human immunodeficiency virus, influenza and respiratory syncytial virus (Fouda et al., 2011; Mazur et al., 2019; Demers-Mathieu et al., 2021b). By the way, the levels of Abs produced in breast milk after COVID-19 mRNA vaccination were significantly higher than those in the natural infection or convalescent period (Low et al., 2021a). Thus, even for lactating women who are naturally infected with COVID-19, vaccination may be helpful to induce and promote the transfer of anti-SARSCoV-2 Abs in breast milk, as these protective Abs acquired after infection will weaken over time. Interestingly, unlike the significant anti-SARS-CoV-2 sIgA levels detected in breast milk after previous infections (Fox et al., 2020), only a few post-vaccination (mRNA-1273 and BNT162b2) milk samples contain spike-specific secretions and the titer is very low (Fox et al., 2021). It is mainly because the intramuscular (IM) vaccination may not elicit a robust sIgA response. Besides, although plasma levels of anti-SARS-CoV-2 IgG, IgM and IgA were not detectable in infants after maternal vaccination during lactation, breastmilk anti-SARS-CoV-2 IgA and IgG survived gastric digestion (Pieri et al., 2021), and notably, anti-SARSCoV-2 IgG was detected in infant stool samples collected 4 and 8 weeks post maternal vaccine and were correlated with maternal milk anti-SARS-CoV-2 IgG levels (Golan et al., 2021c). Thus, milk-derived Abs might persist and provide mucosal immunity in the infant gastrointestinal tract.

By the way, spike-reactive $\mathrm{T}$ cells were also detected in the breast milk of vaccinated mothers (Gonçalves et al., 2021). The frequency seems low, but a suckling infant ingests a lot of milk daily, which implies the ingestion of quantities of spike-reactive T cells. As milk lymphocytes are capable of withstanding the gastric environment, infiltrating the gut mucosa, entering blood circulation and being distributed into infant tissues, further studies are needed to investigate whether milk transferred spike-reactive $\mathrm{T}$ cells could mediate protection against viral infection in the infant upper respiratory tract and gut.

Other ingredients in breast milk that protect against COVID-19 Many bioactive molecules (such as LF, breast milk oligosaccharides, lactadherin, anti-protease and antioxidant factors, etc.) could provide passive protection to the baby. Among them, LF is widely distributed in milk, colostrum and most exocrine secretions that bathe mucosal surfaces. Recently, it has been considered to be one of the bioactive 
inhibitors for SARS-CoV-2 (Mattar et al., 2021) and causes extensive research. Naidu et al. (2020) suggested LF's charge neutralization ability may also act as blocking viral adhesion to the proteoglycan-rich host cell surface (Naidu et al., 2020). Moreover, the interaction between the virus and the host can be interfered by it without internalization in the cell, which is especially effective in the throat, salivary glands, and upper respiratory tract in the early expansion phase of the virus. For example, heparan sulfate proteoglycans (HSPGs) have been proved to be a necessary co-factor for SARS-CoV-2 infection and promote specific attachment to ACE2 receptors and the internalization of the virion ( $\mathrm{Hu}$ et al., 2021). LF can bind to them on the cell surface and prevent infection by blocking the interaction between SARS-CoV-2 and HSPGs. Besides, LF could also selectively inhibit cathepsin L, a lysosomal peptidase critical for endocytosis and a cell entry pathway used by SARS-CoV-2.

In addition to the interaction with host cells, LF can also modulate the immune response. On one hand, oral administration can improve the generation of $\mathrm{CD}+3, \mathrm{CD}+4$, and $\mathrm{CD}+8$ lymphocytes population, and enhance $\mathrm{T}$ cell cytotoxic activities, natural killer cell cytotoxicity, and serum cytokine levels (Kawakami et al., 2015). On the other hand, excessive inflammation could be suppressed by LF. As well known, the virus could cause the propagation of the proinflammatory state, and LF in breast milk not only to promote the differentiation of CD4+ $\mathrm{T}$ cells into Th1 cells to block many inflammatory cytokines such as IL-1 $\beta$, IL-6 and TNF- $\alpha$, but also to stimulate pro-inflammatory macrophages to shift toward anti-inflammatory macrophages.

From the above results, these intrinsic functional properties make maternal-LF a potent innate defense factor to prevent the spread of COVID-19 from mother to newborn, and may also explain why infants have low COVID-19 morbidity and mortality. In addition, given its wide availability, limited cost, and lack of adverse effects, LF could be suggested as both a non-toxic health eement to prevent infection and an adjunct therapy for those who have developed COVID-19. However, more research to verify the dosage and efficacy is still needed.

\section{Limitations}

There are limitations to this review. Firstly, we excluded nonEnglish language studies. Secondly, our review only included data from mRNA vaccines. Several other COVID-19 vaccines remain under investigation.

\section{Conclusion}

Although SARS-CoV-2 RNA has been detected in breast milk, the rate is extremely low and the samples are almost impossible to contain viable viral particles. This seems to invalidate the hypothesis that the virus could be transmitted via maternal milk. Moreover, vaccine mRNA is not present in milk samples, or is only detected in very low levels in some cases. Similarly, the PEG was not found at significant levels in milk after vaccination. These results, consistent with no significant side effects noted in breastfeeding infants, support the safety of vaccination during lactation.

Breast milk of lactating women after infection or mRNA COVID-19 vaccination contains various bioactive molecules like Abs and LF. They can prevent infants' airways and gastrointestinal tract from infections. Interestingly, breast milk after vaccination contains a majority of IgG, whereas an IgA-dominated response was observed in the breast milk of infected women. This difference may be ascribed to the various exposure routes of lactating women to viral antigens. In addition, LF could help infants modulate the overactive inflammatory response of the gastrointestinal caused by SARS-CoV-2 and counteract the activation of the cytokine-storm. Therefore, provided that precautions (wearing masks during breastfeeding, sterilizing breast pumps and washing hands frequently with soap and water, etc.) are taken to avoid spreading the virus to the infants, infected and vaccinated lactating women should continue to breastfeed their infants uninterruptedly to enhance infants' immunity and prevent severe adverse symptoms of SARS$\mathrm{CoV}-2$ infection.

Authors' Contribution: The authors confirm contribution to the paper as follows: Fei Chen designed the original idea and wrote the original draft; Chun Luan, Zichun Wei, Dechen Cai, Zhiwen Cui and Yuyang Li contributed to the bibliographic search and in the original draft writing. Hao $\mathrm{Wu}$, Xiaoxia Zhang and Xiaoli $\mathrm{Wu}$ edited and reviewed the final version of the manuscript. All authors have approved the final version of the manuscript.

Funding Statement: This work was supported by the Innovation and Entrepreneurship Training Program for College Students of Jining Medical University (Grant No. cx2021062) and Natural Science Foundation of Shandong Province (Grant No. ZR2020QC100).

Conflicts of Interest: The authors declare that they have no conflicts of interest to report regarding the present study.

\section{References}

Baird JK, Jensen SM, Urba WJ, Fox BA, Baird JR (2021). SARS-CoV-2 antibodies detected in mother's milk post-vaccination. Journal of Human Lactation 37: 492-498. DOI 10.1177/ 08903344211030168.

Bertino E, Moro GE, De Renzi G, Viberti G, Cavallo R et al. (2020). Detection of SARS-CoV-2 in milk from COVID-19 positive mothers and follow-up of their infants. Frontiers in Pediatrics 8: 597699. DOI 10.3389/fped.2020.597699.

Chambers C, Krogstad P, Bertrand K, Contreras D, Tobin NH, Bode L, Aldrovandi G (2020). Evaluation for SARS-CoV-2 in breast milk from 18 infected women. Journal of the American Medical Association 324: 1347-1348. DOI 10.1001/jama.2020.15580.

Chen H, Guo J, Wang C, Luo F, Yu X et al. (2020). Clinical characteristics and intrauterine vertical transmission potential of COVID-19 infection in nine pregnant women: A retrospective review of medical records. Lancet 395: 809815. DOI 10.1016/S0140-6736(20)30360-3.

Demers-Mathieu V, Do DM, Mathijssen GB, Sela DA, Seppo A, Jarvinen KM, Medo E (2021a). Difference in levels of SARS-CoV-2 S1 and S2 subunits- and nucleocapsid protein-reactive SIgM/IgM, IgG and SIgA/IgA antibodies in human milk. Journal of Perinatology 41: 850-859. DOI 10.1038/s41372-020-00805-w. 
Demers-Mathieu V, Huston RK, Markell AM, McCulley EA, Martin RL, Dallas DC (2021b). Impact of pertussis-specific IgA, IgM, and IgG antibodies in mother's own breast milk and donor breast milk during preterm infant digestion. Pediatric Research 89: 1136-1143. DOI 10.1038/s41390-020-1031-2.

Dong Y, Mo X, Hu Y, Qi X, Jiang F, Jiang Z, Tong S (2020). Epidemiological characteristics of 2143 pediatric patients with 2019 coronavirus disease in China. Journal of Emergency Medicine 58: 712-713. DOI 10.1016/j.jemermed.2020.04.006.

Fenizia C, Biasin M, Cetin I, Vergani P, Mileto D et al. (2020). Analysis of SARS-CoV-2 vertical transmission during pregnancy. Nature Communications 11: 5128. DOI 10.1038/s41467-020-18933-4.

Fouda GG, Yates NL, Pollara J, Shen X, Overman GR et al. (2011). HIV-specific functional antibody responses in breast milk mirror those in plasma and are primarily mediated by IgG antibodies. Journal of Virology 85: 9555-9567. DOI 10.1128/JVI.05174-11.

Fox A, Marino J, Amanat F, Krammer F, Hahn-Holbrook J, ZollaPazner S, Powell RL (2020). Robust and specific secretory IgA against SARS-CoV-2 detected in human milk. iScience 23: 101735. DOI 10.1016/j.isci.2020.101735.

Fox A, Norris C, Amanat F, Zolla-Pazner S, Powell RL (2021). The vaccine-elicited immunoglobulin profile in milk after COVID-19 mRNA-based vaccination is IgG-dominant and lacks secretory antibodies. medRxiv 14: 215. DOI 10.1101/ 2021.03.22.21253831.

Friedman MR, Kigel A, Bahar Y, Yogev Y, Dror Y et al. (2021). BNT162b2 COVID-19 mRNA vaccine elicits a rapid and synchronized antibody response in blood and milk of breastfeeding women. medRxiv. DOI 10.1101/ 2021.03.06.21252603.

Gao X, Wang S, Zeng W, Chen S, Wu J, Lin X, Liu Y, Sun Z, Feng L (2020). Clinical and immunologic features among COVID19-affected mother-infant pairs: Antibodies to SARS-CoV-2 detected in breast milk. New Microbes and New Infections 37: 100752. DOI 10.1016/j.nmni.2020.100752.

Garvey LH, Nasser S (2021). Anaphylaxis to the first COVID-19 vaccine: Is polyethylene glycol (PEG) the culprit? British Journal of Anaesthesia 126: e106-e108. DOI 10.1016/j. bja.2020.12.020.

Golan Y, Prahl M, Cassidy A, Lin CY, Ahituv N, Flaherman VJ, Gaw SL (2021a). COVID-19 mRNA vaccine is not detected in human milk. medRxiv. DOI 10.1101/2021.03.05.21252998.

Golan Y, Prahl M, Cassidy A, Lin CY, Ahituv N et al. (2021b). Evaluation of messenger RNA from COVID-19 BTN162b2 and mRNA-1273 vaccines in human milk. JAMA Pediatrics 175: 1069-1071. DOI 10.1001/jamapediatrics.2021.1929.

Golan Y, Prahl M, Cassidy AG, Gay C, Wu AHB et al. (2021c). COVID-19 mRNA vaccination in lactation: Assessment of adverse events and vaccine related antibodies in motherinfant dyads. medRxiv. DOI 10.3389/fimmu.2021.777103.

Gonçalves J, Juliano AM, Charepe N, Alenquer M, Athayde D, Ferreira F, Archer M, João Amorim M, Serrano F, Soares H (2021). Non-neutralizing secretory IgA and T cells targeting SARS-CoV-2 spike protein are transferred to the breastmilk upon BNT162b2 vaccination. medRxiv. DOI 10.1101/2021.05.03.21256416.

Gray KJ, Bordt EA, Atyeo C, Deriso E, Akinwunmi B et al. (2021). COVID-19 vaccine response in pregnant and lactating women: A cohort study. medRxiv 39: 868. DOI 10.1101/ 2021.03.07.21253094.
Hu Y, Meng X, Zhang F, Xiang Y, Wang J (2021). The in vitro antiviral activity of lactoferrin against common human coronaviruses and SARS-CoV-2 is mediated by targeting the heparan sulfate co-receptor. Emerging Microbes \& Infections 10: 317330. DOI 10.1080/22221751.2021.1888660.

Hurley WL, Theil PK (2011). Perspectives on immunoglobulins in colostrum and milk. Nutrients 3: 442-474. DOI 10.3390/ nu3040442.

Jakuszko K, Kościelska-Kasprzak K, Żabińska M, Bartoszek D, Poznański P, Rukasz D, Kłak R, Królak-Olejnik B, Krajewska M (2021). Immune response to vaccination against COVID-19 in breastfeeding health workers. Vaccines 9: 663. DOI 10.3390/vaccines9060663.

Kawakami H, Park H, Park S, Kuwata H, Shephard RJ, Aoyagi Y (2015). Effects of enteric-coated lactoferrin supplementation on the immune function of elderly individuals: A randomised, double-blind, placebo-controlled trial. International Dairy Journal 47: 79-85. DOI 10.1016/j.idairyj.2015.02.001.

Kilic T, Kilic S, Berber NK, Gunduz A, Ersoy Y (2021). Investigation of SARS-CoV-2 RNA in milk produced by women with COVID-19 and follow-up of their infants: A preliminary study. International Journal of Clinical Practice 75: e14175. DOI 10.1111/ijcp.14175.

Kunjumon B, Wachtel EV, Lumba R, Quan M, Remon J et al. (2021). Breast milk and breastfeeding of infants born to SARS-CoV-2 positive mothers: A prospective observational cohort study. American Journal of Perinatology 38: 1209-1216. DOI 10.1055/s-0041-1731451.

Lechosa-Muñiz C, Paz-Zulueta M, Mendez-Legaza JM, IrureVentura J, Cuesta González R et al. (2021). Induction of SARS-CoV-2-Specific IgG and IgA in serum and milk with different SARS-CoV-2 vaccines in breastfeeding women: A cross-sectional study in Northern Spain. International Journal of Environmental Research and Public Health 18: 8831. DOI 10.3390/ijerph18168831.

Liu W, Wang J, Li W, Zhou Z, Liu S, Rong Z (2020). Clinical characteristics of 19 neonates born to mothers with COVID-19. Frontiers of Medicine 14: 193-198. DOI 10.1007/s11684-020-0772-y.

Low JM, Gu Y, Ng MSF, Amin Z, Lee LY et al. (2021a). BNT162b2 vaccination induces SARS-CoV-2 specific antibody secretion into human milk with minimal transfer of vaccine mRNA. medRxiv. DOI 10.1101/2021.04.27.21256151.

Low JM, Gu Y, Ng MSF, Amin Z, Lee LY et al. (2021b). Codominant IgG and IgA expression with minimal vaccine mRNA in milk of BNT162b2 vaccinees. npj Vaccines 6: 105. DOI 10.1038/ s41541-021-00370-z.

Low JM, Lee LY, Ng YPM, Zhong Y, Amin Z (2021c). Breastfeeding mother-child clinical outcomes after COVID-19 vaccination. medRxiv. DOI 10.1101/2021.1106.1119.21258892.

Mattar EH, Elrashdy F, Almehdar HA, Uversky VN, Redwan EM (2021). Natural resources to control COVID-19: Could lactoferrin amend SARS-CoV-2 infectivity? PeerJ 9: e11303. DOI 10.7717/peerj.11303.

Mazur NI, Horsley NM, Englund JA, Nederend M, Magaret A et al. (2019). Breast milk prefusion $F$ immunoglobulin $G$ as a correlate of protection against respiratory syncytial virus acute respiratory illness. Journal of Infectious Diseases 219: 59-67.

McLaurin-Jiang S, Garner CD, Krutsch K, Hale TW (2021). Maternal and child symptoms following COVID-19 vaccination among breastfeeding mothers. Breastfeeding Medicine 16: 702-709. DOI 10.1089/bfm.2021.0079. 
Naidu SAG, Clemens RA, Pressman P, Zaigham M, Davies KJA, Naidu AS (2020). COVID-19 during pregnancy and postpartum. Journal of Dietary Supplements, 1-37. DOI 10.1080/19390211.2020.1834047.

Palmeira P, Carneiro-Sampaio M (2016). Immunology of breast milk. Revista Da Associacao Medica Brasileira 62: 584-593. DOI 10.1590/1806-9282.62.06.584.

Perl SH, Uzan-Yulzari A, Klainer H, Asiskovich L, Youngster M, Rinott E, Youngster I (2021). SARS-CoV-2-specific antibodies in breast milk after COVID-19 vaccination of breastfeeding women. Journal of the American Medical Association 325: 2013-2014. DOI 10.1001/jama.2021.5782.

Pieri M, Nicolaidou V, Paphiti I, Pipis S, Felekkis K et al. (2021). Survival of vaccine-induced human milk SARS-CoV-2 IgG and IgA immunoglobulins across simulated human infant gastrointestinal digestion. medRxiv. DOI 10.1101/ 2021.1106.1117.21259021.

Riphagen S, Gomez X, Gonzalez-Martinez C, Wilkinson N, Theocharis P (2020). Hyperinflammatory shock in children during COVID-19 pandemic. Lancet 395: 1607-1608. DOI 10.1016/S0140-6736(20)31094-1.

Robertson CA, Lowther SA, Birch T, Tan C, Sorhage F, Stockman L, McDonald C, Lingappa JR, Bresnitz E (2004). SARS and pregnancy: A case report. Emerging Infectious Diseases 10: 345-348. DOI 10.3201/eid1002.030736.

Sahin D, Tanacan A, Erol SA, Anuk AT, Eyi EGY et al. (2020). A pandemic center's experience of managing pregnant women with COVID-19 infection in Turkey: A prospective cohort study. International Journal of Gynecology \& Obstetrics 151: 74-82. DOI 10.1002/ijgo.13318.

Sahin D, Tanacan A, Erol SA, Anuk AT, Yetiskin FDY et al. (2021). Updated experience of a tertiary pandemic center on
533 pregnant women with COVID-19 infection: A prospective cohort study from Turkey. International Journal of Gynecology \& Obstetrics 152: 328-334. DOI 10.1002/ijgo.13460.

Sellaturay P, Nasser S, Ewan P (2021). Polyethylene glycol-induced systemic allergic reactions (Anaphylaxis). Journal of Allergy and Clinical Immunology 9: 670-675.

Selma-Royo M, Bäuerl C, Mena-Tudela D, Aguilar-Camprubí L, Pérez-Cano FJ (2021). Anti-SARS-CoV-2 IgA and IgG in human milk after vaccination is dependent on vaccine type and previous SARS-CoV-2 exposure: A longitudinal study. medRxiv. DOI 10.1101/2021.05.20.21257512.

Tang A, Tong ZD, Wang HL, Dai YX, Li KF et al. (2020). Detection of novel coronavirus by RT-PCR in stool specimen from asymptomatic child. China Emerging Infectious Diseases 26: 1337-1339. DOI 10.3201/eid2606.200301.

Unger S, Christie-Holmes N, Guvenc F, Budylowski P, Mubareka S, Gray-Owen SD, O'Connor DL (2020). Holder pasteurization of donated human milk is effective in inactivating SARSCoV-2. Canadian Medical Association Journal 192: E871E874. DOI 10.1503/cmaj.201309.

van der Strate BW, Beljaars L, Molema G, Harmsen MC, Meijer DK (2001). Antiviral activities of lactoferrin. Antiviral Research 52: 225-239. DOI 10.1016/S0166-3542(01)00195-4.

Verdoni L, Mazza A, Gervasoni A, Martelli L, Ruggeri M, Ciuffreda M, Bonanomi E, D’Antiga L (2020). An outbreak of severe Kawasaki-like disease at the Italian epicentre of the SARSCoV-2 epidemic: An observational cohort study. Lancet 395: 1771-1778. DOI 10.1016/S0140-6736(20)31103-X.

World Health Organization (2021). WHO Coronavirus Disease (COVID-19) Dashboard. https://covid19.who.int/. 